\title{
Study of In-Patient Cost Analysis using Hospital Database in Korea
}

\author{
Woo Jung-Sik ${ }^{1}$ and Kim Han-Sung* $*^{2}$ \\ ${ }^{1}$ Dept. of Health Administration, Cheju Halla University, Republic of Korea \\ ${ }^{2}$ Dept. of Healthcare Informatics, Korea Polytechnics, Republic of Korea \\ ${ }^{1}$ jswoo@chu.ac.kr, ${ }^{2}$ khs0113@kopo.ac.kr
}

\begin{abstract}
This study is to figure out Influence factors on Admission costs, based on In-Patient Cost Analysis. The study reviewed German Cost Analysis Method, drew Cost analysis Method applicable to Korean hospitals, and then conducted Analysis of Variance (ANOVA) and Multiple Regression, to analyze what factors influenced Admission Costs. The empirical analysis has shown that the cost recovery level was influenced by 'Hospital visit days', 'Examination costs' and 'Physician costs' Especially the cost recovery level had a positive relationship with Examination cost rate and a negative relationship with Physician cost rate, which directly affected surgical units and medical units thus caused the imbalance of Cost recovery level between both units. In other words, surgical units cost recovery level was low, however medical units was relatively high. Therefore the improvement of Cost recovery system, based on the exact cost information is needed to solve the imbalance of cost recovery level.
\end{abstract}

Keywords: Hospital Cost; Cost Accounting System; Admission Cost; Cost Center

\section{Introduction}

The cost recovery method of Korean National Health Insurance for Physicians medical service basically stands on Fee for Services, and has gone through three big changes. When introducing Medical Insurance for the first time in 1977, it adopted Point System but it was changed to Amount System which is the system announcing each service cost, in 1981. And in 2001, Resource based Relative Value Scale (RBRVS) on the basis of Point for services and Conversion Factor was introduced, and has been continued until now.

Medical Insurance Fee system used when introducing was lack of objective bases, because it referred already customized or other countries fee. Moreover, it had been criticized that each service input cost was imbalanced.

Relative Value Scale (RBRVS) is based on Resource use for services. It is the way which can seek not only the differential of resources relative value but also the suitability of insurance fee, and it has a strong point to be able to guarantee more objective bases than the existing systems such as the Amount System, when measuring the value of service [1]. Korean Relative Value Scale accounts Resource use for services, on the basis of Physician workload, Medical service costs (Labor costs for Medical staffs, Material costs, Equipment costs, and risk grade.

Now it is under the circumstance that hospitals targeted cost analysis is very rare, even though Medical service suppliers are expressing their economic difficulties, caused by low recovery rate for insured Medical service costs. That's why this study is to review German cost accounting method, to produce Patient Cost Accounting Method with ABC cost analysis system applied, and then to present the difference between Patient cost and Cost recovery level, and Influence factors on Cost recovery level. 


\section{Theoretical Consideration}

Generally speaking, Cost is the amount measuring tangible or intangible value which is given up spontaneously or by force, for the acquisition or the creation of economic value. And hospital cost is to express economic value of goods and services, consumed in the process of medical service for patients. The economic value includes not only direct medical service costs but also Assistant and Maintenance costs for supporting direct medical services. That is, Cost is the flow of economic value, used in the process of Hospital Management and Cost Accounting is to classify and to account Labor costs, Material costs and Maintenance costs and etc.. by Cost unit, and then to sum up them by place occurred.

The reasons why Cost Accounting is needed in hospitals are as follows. First, it is to develop the reasonable fee for Health Insurance Policy. At the moment, Korean Fee is decided by 'RBRVS' and 'Conversion Factor' and is benchmarking SGR (Sustainable Growth Rate) of USA for its adjustment. However SGR model considers only the inflation rate for next year, compared to the base year on several macroeconomic indicators, and doesn't care about medical service needed resources. So the medical service suppliers are complaining about that the cost recovery doesn't work properly, and are continuously asking the reorganization of medical fee system. Second, it can improve the efficiency of hospital management by collecting cost information. It is possible to figure out resource input versus consumption by systematic cost accounting, furthermore to confirm detailed element status. These information will be very useful to allocate or reassign resources.

But for hospital cost accounting, it is difficult to choose the objects of cost, and even if it can choose, it is very difficult to apply them collectively because resource input kinds are various by object and the volume is not fixable [2]. In other words, not only hospital cost's allocation process is complicated, but also it's not easy to divide 'Common expenses' into each service. From the reason, studies of 'Hospital cost' are not many, moreover patient cost analysis with which this study is to deal, has not been studied at all.

European countries where hospital cost analysis is being studied lively, have tried to enhance the accuracy of cost accounting to maximize the merits of DRG [3]. They thought that the exact understanding of cost must be considered above all, for serving the effective and fair medical services. Under this understanding, many countries such as England, France, Germany and etc.. have chosen standard hospitals, have collected Cost information regularly and have developed DRG system based on them.

In theory, Cost has to be allocated to all patients who are homogeneous medically and in cost-related [4-5]. In case of German where Patient cost accounting system is relatively well built up, all hospitals account Cost by cost-center depending on hospital accounting standards, which has been conducted initiatively by InEK (Institute for the Hospital Remuneration System). German's DRG Cost Matrix System structurizes cost items (Labor costs, Material costs and Infrastructure costs), cost-center group, and allocation scheme, being utilized for patient cost accounting [6].

Many european countries are using Cost data to develop DRG system. Among those countries, all hospitals of England, Portugal and Netherlands are building up mandatory cost-accounting system and annually reporting data from it to their governments. And some countries like Germany, France, Ireland and Sweden which don't take the system are in accordance with National costing guidelines. If taking a look into Cost allocation method of these countries, common expenses are allocated to each medical department by Direct cost allocation to patients (England, Estonia, Finland, Netherlands and Sweden), or Indirect cost allocation to patients (France and Germany). Those expenses which are allocated to each medical department are again allocated to each patient. And many countries except for Austria apply weighting statistics to Micro-costing accounting [7]. 
The first verification of Cost data is up to hospitals. The central government in case of England, Estonia and Netherlands, and local authorities in Austria and France do the second. Finland is exceptional. In Finland, Cost data is not supervised by the central government or local authority systematically, so hospitals take all responsibilities [8].

\section{Analysis Method}

\subsection{Objects of Study}

This study analyzed cost with German cost structure applied. The basic cost allocation method adopted complete cost allocation method which allocates total expenses for each in-patient who got service in cost-center. The basic data for Cost analysis are from 2010 data of a general hospital which manages with 'Activity-based cost analysis system' and the final Cost recovery rate was calculated by connecting 'Patient cost' to Health Insurance Medical Service cost data.

Also to calculate Patient cost, each expense data of Labor costs(doctors and medical staff costs differentiated) by cost-center group, Material costs, Equipment costs and Management costs was collected. Cost-center consisted of 136 units, and each cost-center included all occurred medical record data, regardless of the kinds of Insurer(National health insurance, Medicaid, Auto insurance, Worker's compensation insurance and General patients). As the result, 24,646 Patients medical fees were analyzed with excluding unstable data $\langle$ Table 1$\rangle$.

Table 1. Characteristics of the Objects of Analysis

\begin{tabular}{|c|c|c|c|}
\hline \multicolumn{2}{|c|}{ Classification } & Frequency $(\mathrm{N})$ & Ratio(\%) \\
\hline \multirow[t]{9}{*}{ Age } & 0 & 976 & 4.0 \\
\hline & Age 1-10 & 2,860 & 11.6 \\
\hline & Age $11-20$ & 1,026 & 4.2 \\
\hline & Age $21-30$ & 1,334 & 5.4 \\
\hline & Age $31-40$ & 2,246 & 9.1 \\
\hline & Age 41-50 & 3,110 & 12.6 \\
\hline & Age $51-60$ & 3,140 & 12.7 \\
\hline & Age 61-70 & 4,616 & 18.7 \\
\hline & More than 70 & 5,338 & 21.7 \\
\hline \multirow[t]{2}{*}{ Gender } & Male & 12,514 & 50.8 \\
\hline & Female & 12,132 & 49.2 \\
\hline \multirow[t]{6}{*}{ Hospital visit days } & 1-15 days & 21,012 & 85.3 \\
\hline & 16-30 days & 2,455 & 10.0 \\
\hline & 31-45 days & 560 & 2.3 \\
\hline & 46-60 days & 261 & 1.1 \\
\hline & $61-75$ days & 191 & 0.8 \\
\hline & More than 75 & 167 & 0.7 \\
\hline \multirow[t]{11}{*}{ Medical departments } & Internal Medicine & 8,957 & 36.3 \\
\hline & Neurology & 879 & 3.6 \\
\hline & Psychiatrics & 783 & 3.2 \\
\hline & General Surgery & 1,462 & 5.9 \\
\hline & Orthopedics & 2,386 & 9.7 \\
\hline & Neurosurgery & 999 & 4.1 \\
\hline & Cardiothoracic Surgery & 532 & 2.2 \\
\hline & Plastic Surgery & 201 & 0.8 \\
\hline & $\mathrm{Ob} \& \mathrm{~Gy}$ & 795 & 3.2 \\
\hline & Pediatrics & 3,590 & 14.6 \\
\hline & Ophthalmology & 235 & 1.0 \\
\hline
\end{tabular}




\begin{tabular}{c|c|c|c} 
& Otolaryngology & 957 & 3.9 \\
& Urology & 891 & 3.6 \\
& Rehabilitation & 298 & 1.2 \\
& Family Medicine & 973 & 3.9 \\
& Emergency Medicine & 574 & 2.3 \\
& Other Assistant departments & 134 & 0.5 \\
\hline Operation & Non-operation & 19,016 & 77.2 \\
& Operation & 5,630 & 22.8 \\
\hline Emergency room & Non-use & 15,971 & 64.8 \\
visit & Use & 8,675 & 35.2 \\
\hline Classification of & Simple DRG & 3,818 & 15.5 \\
DRG & General DRG & 16,738 & 67.9 \\
& Special DRG & 4,090 & 16.6 \\
\hline Total Medical Costs & Less than 510,000 & 5,658 & 23.0 \\
(Korean Won) & $510,000-1,000,000$ & 6,787 & 27.5 \\
& $1,010,000-1,500,000$ & 3,439 & 14.0 \\
& $1,510,000-2,000,000$ & 2,173 & 8.8 \\
& $2,010,000-2,500,000$ & 1,286 & 5.2 \\
& $2,510,000-5,000,000$ & 2,909 & 11.8 \\
& More than 5,000,000 & 2,394 & 9.7 \\
\hline & Total & 24,646 & 100.0 \\
\hline
\end{tabular}

\section{The Process of Cost Analysis}

\subsection{Cost-center Setting}

Cost center is the first aggregation unit of the occurred cost, and there's no consistent theory of Cost center setting method. Generally speaking, Cost center is decided by the scale, characteristics and the structure of an organization, and the more they are subdivided, the higher the efficiency of cost analysis goes. But the more expenses are also needed [9]. If cost centers are not many on the contrary, it is likely that inhomogeneous items are possible to be considered as one [10]. Expecting that the enough experience is able to cover such different views, this study derived Cost analysis model applicable to Korean circumstance, based on German cost analysis model.

\subsection{The Classification of Cost Units}

Cost units consist of 'Labor costs', 'Material costs' and 'Maintenance costs'. 'Labor costs' are related to the labor, required in the process of medical profit creation and cover all kinds of wage paid to all members. The most part of Hospital labor costs is paid to Physicians who give medical services and clinical human resources(medical technicians and $e t c .$.$) . This study subdivided labor costs into Physicians, clinical human resources and$ their assistants.

'Material costs' are divided into separate compensation provided material costs(the costs which can be charged to insurer) and non-separate compensation provided material costs(the costs which can't be charged to insurer on account of that the cost is already included in medical service). Patient cost was aggregated when a separate compensation was done because the compensation was done on a basis of market price. For nonseparate compensation provided material costs on the other hand, the total occurred amount for a year was allocated per Patient, by allocation scheme.

'Maintenance costs' include all expenses except for Labor costs and 'Material costs' among expenditures for Hospital's medical profit creation activity. 'Maintenance costs' were allocated by each cost center unit activity. However depreciation costs for medical 
devices and Maintenance costs were treated as Direct costs, owing to their direct cost-like characteristics in this study.

\subsection{In-Patient Cost Allocation}

German cost accounting method was referred for In-patient cost allocation. 'In-patient room' costs were calculated by dividing total expenses from In-patients wards into the sum of all patients admission days(one patient cost), next by multiplying the sum and each patient's admission days.

For 'Operating rooms' and 'Anesthesia rooms', it applied Expenses per minute to a patient's operation time and anesthesia time. In case of 'Psycho theraphy rooms', 'Physical theraphy rooms' and 'Laboratories', the input resources depended on what kind of examinations were done, so the total Relative value scores(Relative value score $\times$ Annual examination numbers) by Examination were applied to Point cost accounting. And about Extracorporeal Shock Wave Liythoripsy(ESWL) rooms, the numbers of ESWL were accounted and the weight was applied; $100 \%$ for 1 time, $50 \%$ for $2-5$ and $25 \%$ for over 6. 'Hemodialysis' rooms were calculated with Dialysis costs per minute, using the total dialysis time.

The In-patient cost unit allocation model is as <Table 2>. When setting up such an allocation scheme, The National Health Insurance data were used subsidiarily in case there were no direct data. The data such as Delivery numbers, Intensive Care Unit(ICU) Using days and the accumulated patient numbers for Emergency room were input as proxy variables, related to Delivery wards, Intensive Care Units and Emergency rooms. Besides, $80 \%$ of anesthesia time was accounted in case of non-accountable operation time, and Relative Value Score for ESWL rooms and the numbers of dialysis for Hemodialysis rooms were used as Scheme data. 
Table 2. Model of Hospital Inpatient Cost Matrix

\begin{tabular}{|c|c|c|c|c|c|c|c|c|}
\hline \multirow{2}{*}{\multicolumn{2}{|c|}{$\begin{array}{l}\text { Cost } \\
\text { Cost } \\
\text { Center Group }\end{array}$}} & \multicolumn{2}{|c|}{ Labor costs } & \multicolumn{2}{|c|}{ Material costs } & \multirow{2}{*}{$\begin{array}{l}\text { Drugs } \\
\text { costs }\end{array}$} & \multirow{2}{*}{$\begin{array}{l}\text { Equipment } \\
\text { costs }\end{array}$} & \multirow{2}{*}{$\begin{array}{l}\text { Maintenance } \\
\text { costs }\end{array}$} \\
\hline & & Physicians & $\begin{array}{c}\text { Medical } \\
\text { staff }\end{array}$ & $\begin{array}{c}\text { Separate } \\
\text { compensation }\end{array}$ & $\begin{array}{l}\text { Include } \\
\text { services }\end{array}$ & & & \\
\hline \multirow{4}{*}{ Ward } & Delivery ward & \multicolumn{2}{|c|}{ Actual time } & \multirow{14}{*}{$\begin{array}{l}\text { Actual } \\
\text { usage } \\
\text { cost }\end{array}$} & Actual time & \multirow{14}{*}{$\begin{array}{l}\text { Actual } \\
\text { usage } \\
\text { cost }\end{array}$} & Actual time & Actual time \\
\hline & $\begin{array}{l}\text { Intensive care } \\
\text { unit }\end{array}$ & \multicolumn{2}{|c|}{ Actual time } & & Actual time & & Actual time & Actual time \\
\hline & General Ward & \multicolumn{2}{|l|}{ Care days } & & Care days & & Care days & Care days \\
\hline & $\begin{array}{l}\text { Emergency } \\
\text { room }\end{array}$ & \multicolumn{2}{|c|}{ Actual time } & & Actual time & & Actual time & Actual time \\
\hline \multirow{2}{*}{$\begin{array}{l}\text { Operating } \\
\text { rooms }\end{array}$} & $\begin{array}{l}\text { Operating } \\
\text { rooms }\end{array}$ & \multicolumn{2}{|c|}{ Operation time } & & $\begin{array}{l}\text { Operation } \\
\text { time }\end{array}$ & & $\begin{array}{l}\text { Operation } \\
\text { time }\end{array}$ & $\begin{array}{l}\text { Operation } \\
\text { time }\end{array}$ \\
\hline & $\begin{array}{l}\text { Anesthesia } \\
\text { (for operation) }\end{array}$ & \multicolumn{2}{|c|}{ Anesthesia time } & & $\begin{array}{l}\text { Anesthesia } \\
\text { time }\end{array}$ & & $\begin{array}{l}\text { Anesthesia } \\
\text { time }\end{array}$ & $\begin{array}{l}\text { Anesthesia } \\
\text { time }\end{array}$ \\
\hline \multicolumn{2}{|c|}{$\begin{array}{l}\text { Anesthesia } \\
\text { (for treatment) }\end{array}$} & \multicolumn{2}{|c|}{ RBRVS Score } & & $\begin{array}{l}\text { RBRVS } \\
\text { Score }\end{array}$ & & $\begin{array}{l}\text { RBRVS } \\
\text { Score }\end{array}$ & $\begin{array}{l}\text { RBRVS } \\
\text { Score }\end{array}$ \\
\hline Radiology & $\begin{array}{l}\text { SPECT, MRI, } \\
\text { CT, X-Ray etc. }\end{array}$ & RBRVS Sc & core & & $\begin{array}{l}\text { RBRVS } \\
\text { Score }\end{array}$ & & $\begin{array}{l}\text { RBRVS } \\
\text { Score }\end{array}$ & $\begin{array}{l}\text { RBRVS } \\
\text { Score }\end{array}$ \\
\hline $\begin{array}{l}\text { Sample } \\
\text { Laboratories }\end{array}$ & $\begin{array}{l}\text { Blood, Urine, } \\
\text { Microorganism } \\
\text { etc. }\end{array}$ & RBRVS Sc & core & & $\begin{array}{l}\text { RBRVS } \\
\text { Score }\end{array}$ & & $\begin{array}{l}\text { RBRVS } \\
\text { Score }\end{array}$ & $\begin{array}{l}\text { RBRVS } \\
\text { Score }\end{array}$ \\
\hline $\begin{array}{l}\text { Functional } \\
\text { Laboratories }\end{array}$ & $\begin{array}{l}\text { Endoscope, } \\
\text { ECG, EEG, } \\
\text { Respiratory } \\
\text { function } \text { etc. }\end{array}$ & RBRVS Sc & core & & $\begin{array}{l}\text { RBRVS } \\
\text { Score }\end{array}$ & & $\begin{array}{l}\text { RBRVS } \\
\text { Score }\end{array}$ & $\begin{array}{l}\text { RBRVS } \\
\text { Score }\end{array}$ \\
\hline \multicolumn{2}{|c|}{$\begin{array}{l}\text { Physical Theraphy / } \\
\text { Psycho Theraphy }\end{array}$} & \multicolumn{2}{|c|}{ RBRVS Score } & & $\begin{array}{l}\text { RBRVS } \\
\text { Score }\end{array}$ & & $\begin{array}{l}\text { RBRVS } \\
\text { Score }\end{array}$ & $\begin{array}{l}\text { RBRVS } \\
\text { Score }\end{array}$ \\
\hline \multicolumn{2}{|c|}{$\begin{array}{l}\text { ESWL(Extracor-poreal } \\
\text { Shock Wave Liythoripsy) }\end{array}$} & \multicolumn{2}{|c|}{ Number of ESWL } & & $\begin{array}{l}\text { Number of } \\
\text { ESWL }\end{array}$ & & $\begin{array}{l}\text { Number of } \\
\text { ESWL }\end{array}$ & $\begin{array}{l}\text { Number of } \\
\text { ESWL }\end{array}$ \\
\hline \multicolumn{2}{|l|}{ Hemodialysis } & \multicolumn{2}{|c|}{ Hemodialysis time } & & $\begin{array}{l}\text { Hemodialysis } \\
\text { time }\end{array}$ & & $\begin{array}{l}\text { Hemodialysis } \\
\text { time }\end{array}$ & $\begin{array}{l}\text { Hemodialysis } \\
\text { time }\end{array}$ \\
\hline \multicolumn{2}{|l|}{ Pharmacy } & \multicolumn{2}{|c|}{ RBRVS Score } & & $\begin{array}{l}\text { RBRVS } \\
\text { Score }\end{array}$ & & $\begin{array}{l}\text { RBRVS } \\
\text { Score }\end{array}$ & $\begin{array}{l}\text { RBRVS } \\
\text { Score }\end{array}$ \\
\hline
\end{tabular}

\section{The Results of Patient Cost Analysis}

\subsection{Cost per Unit}

First of all, the allocation scheme score per cost unit was set up. Table 3 is the scheme for all patients no matter who the patients (National health insurance, Worker's compensation insurance, Medicaid and etc.) are and no matter what kind of services (Inpatient and Outpatient) are. For instance, 829,440 minutes of 'Operating room' include the operation time not only of National health insurance patients but also of Worker's compensation insurance and Medicaid patients, which accumulate all medical service time for In-patients and Out-patients. In addition to that, 204 scores of 'Laboratories' mean the total Relative Value scores of all examination services which Inpatients and 
Outpatients were served for an year. But the data were excluded, if the separate compensation was done or the patient paid $100 \%$ of Medical material costs and Pharmaceutical costs.

Table 3. Allocation Scheme per Cost Unit

\begin{tabular}{|c|c|c|c|c|}
\hline \multicolumn{2}{|c|}{ Cost Unit } & Allocation Scheme & $\begin{array}{l}\text { Total Costs } \\
\text { (Korean Won) }\end{array}$ & $\begin{array}{c}\text { Allocation } \\
\text { Scheme Score }\end{array}$ \\
\hline \multirow{4}{*}{ Ward } & Delivery ward & $\begin{array}{c}\text { Spontaneous Delivery } \\
\text { Number }\end{array}$ & $326,538,370$ & 503 cases \\
\hline & Emergency room & $\begin{array}{c}\text { Accumulated } \\
\text { Emergency Room } \\
\text { Patient Number }\end{array}$ & $5,425,116,136$ & $\begin{array}{l}18,191 \\
\text { Inpatients }\end{array}$ \\
\hline & General Ward & Inpatient Days & $22,317,986,183$ & 285,555 days \\
\hline & Intensive care unit & $\begin{array}{l}\text { Intensive care unit } \\
\text { Inpatient Days }\end{array}$ & $5,138,447,775$ & 18,628 days \\
\hline \multirow{2}{*}{ Operating Rooms } & Operating rooms & Operation Time & $7,688,729,699$ & 829,440 minutes \\
\hline & $\begin{array}{l}\text { Anesthesia(for } \\
\text { operation) }\end{array}$ & Anesthesia Time & $2,317,353,235$ & $\begin{array}{l}1,036,800 \\
\text { minutes }\end{array}$ \\
\hline \multicolumn{2}{|c|}{ Anesthesia Rooms(For treatment) } & RBRVS Score & $310,031,385$ & $3,169,751$ points \\
\hline Laboratories & $\begin{array}{l}\text { Specimen/Function } \\
\text { test }\end{array}$ & RBRVS Score & $11,030,892,492$ & $\begin{array}{l}204,420,665 \\
\text { points }\end{array}$ \\
\hline Radiology & Imaging test & RBRVS Score & $10,209,681,745$ & $\begin{array}{l}154,486,358 \\
\text { points }\end{array}$ \\
\hline Physical/Psycho Theraphy & $\begin{array}{l}\text { Physical/Psycho } \\
\text { Theraphy }\end{array}$ & RBRVS Score & $2,355,732,091$ & $\begin{array}{l}34,486,403 \\
\text { points }\end{array}$ \\
\hline $\begin{array}{l}\text { ESWL(Extracor-poreal } \\
\text { Shock Wave Liythoripsy) }\end{array}$ & ESWL & RBRVS Score & $127,366,580$ & $2,041,452$ points \\
\hline Hemodialysis & Hemodialysis & Hemodialysis Number & $2,048,464,239$ & 26,250 cases \\
\hline Pharmacy & $\begin{array}{l}\text { Medication } \\
\text { preparation }\end{array}$ & RBRVS Score & $3,075,921,969$ & $\begin{array}{l}12,777,902 \\
\text { points }\end{array}$ \\
\hline
\end{tabular}

\subsection{The Difference between Cost Recovery Level (Medical Service Costs), Cost Level and Cost Recovery Rate}

Table 4 shows In-patient characteristics difference between Cost recovery level and Cost level. Though both Cost recovery level and Cost level tended to increase as 'Age' went higher, Cost level was higher than Cost recovery level in all ages. The difference of Cost recovery level was most in 21 30 and gradually decreased from over 30 . The highest Cost recovery rate group was the aged over 70 .

The absolute Cost recovery level for women was lower than for men, but Cost level was higher on the contrary. Cost recovery rate was higher for men and the difference between two was meaningful statistically. As 'Admission days' got longer, the difference between Medical service costs and Cost level gradually increased except for 31-45 days. Cost recovery was highest for 31-45 days and lowest for 64-75 days.

For Medical departments, Cost recovery rate of Internal Medicine(99.8) and Family Medicine(103.8\%) was satisfactory. Cost recovery level of all departments except for Family Medicine was below Cost level. Otolaryngology(69.8\%), Emergency Medicine(72.1\%) and $\mathrm{Ob} / \mathrm{Gy}(73.2 \%)$ was especially low. 
Related to 'Operation', the difference between Cost recovery level and Cost level was big when operating. The Operated group's Cost recovery rate was only $70 \%$, while NonOperated group'was over 93\%. The Cost recovery rate when visiting Emergency room was a bit lower than when not visiting, this was meaningful statistically.

For Diagnosis related group(DRG), Cost recovery level and Cost level of Special DRG were absolutely high, compared to other DRGs(Simple DRG and General DRG). Cost recovery rate also was fine in Special DRG(94.3) than other DRGs(Simple DRG:82.5\%, General DRG:87.7\%). The severity of DRG was similar to DRG classification. The group with high severity had higher Cost recovery rate than the group with low. Especially the Cost recovery was $100 \%$ achieved in the group with grade 3 of severity.

It turned out that the more Medical service costs were, the bigger the difference between total Medical service costs and Cost level was, within 5,000,000 Korean Won of total Medical costs. The Cost recovery rate was more satisfactory as total Medical costs increased.

Table 4. Comparison between Medical Costs by Characteristics and Costs

\begin{tabular}{|c|c|c|c|c|c|c|c|}
\hline \multirow{2}{*}{\multicolumn{2}{|c|}{ Classification }} & \multirow{3}{*}{$\begin{array}{c}\text { Number of } \\
\text { Patients } \\
976\end{array}$} & \multicolumn{3}{|c|}{ Mean (Korean Won) } & \multirow{2}{*}{$\begin{array}{c}\text { Cost recovery } \\
\text { rate } \\
\text { (Mean } \pm \text { Standard } \\
\text { deviation) }\end{array}$} & \multirow[b]{2}{*}{ Statistic } \\
\hline & & & Medical & & Difference & & \\
\hline \multirow[t]{9}{*}{ Age } & 0 & & 610,419 & 761,289 & 150,870 & $80.1 \pm 13.4$ & \multirow{9}{*}{$\mathrm{F}=281.29 * *$} \\
\hline & $1-10$ & 2,860 & 668,863 & 799,069 & 130,207 & $81.8 \pm 18.1$ & \\
\hline & $11-20$ & 1,026 & $1,153,071$ & $1,424,177$ & 271,106 & $80.3 \pm 21.8$ & \\
\hline & $21-30$ & 1,334 & $1,193,442$ & $1,543,225$ & 349,783 & $78.7 \pm 22.9$ & \\
\hline & $31-40$ & 2,246 & $1,487,182$ & $1,809,901$ & 322,719 & $81.5 \pm 26.6$ & \\
\hline & $41-50$ & 3,110 & $1,927,975$ & $2,223,434$ & 295,459 & $84.9 \pm 21.8$ & \\
\hline & $51-60$ & 3,140 & $2,231,995$ & $2,474,775$ & 242,781 & $89.2 \pm 20.8$ & \\
\hline & $61-70$ & 4,616 & $2,696,897$ & $2,892,350$ & 195,453 & $92.8 \pm 19.2$ & \\
\hline & More than 70 & 5,338 & $2,861,146$ & $2,936,380$ & 75,234 & $96.1 \pm 18.5$ & \\
\hline \multirow[t]{2}{*}{ Gender } & Male & 12,514 & $2,015,263$ & $2,195,588$ & 180,325 & $88.5 \pm 21.4$ & \multirow{2}{*}{$\mathrm{T}=17.14 * *$} \\
\hline & Female & 12,132 & $1,989,051$ & $2,213,059$ & 224,008 & $87.4 \pm 21.1$ & \\
\hline \multirow{6}{*}{$\begin{array}{l}\text { Hospital } \\
\text { visit days }\end{array}$} & 1-15 days & 21,012 & $1,265,206$ & $1,441,083$ & 175,877 & $86.9 \pm 21.5$ & \multirow{6}{*}{$\mathrm{F}=81.53 * *$} \\
\hline & 16-30 days & 2,455 & $4,795,767$ & $5,117,791$ & 322,024 & $94.3 \pm 17.8$ & \\
\hline & 31-45 days & 560 & $6,827,734$ & $6,996,767$ & 169,032 & $96.9 \pm 16.4$ & \\
\hline & 46-60 days & 261 & $8,842,756$ & $9,178,523$ & 335,766 & $94.6 \pm 18.7$ & \\
\hline & $61-75$ days & 191 & $9,753,833$ & $10,608,577$ & 854,744 & $86.2 \pm 29.3$ & \\
\hline & More than 75 days & 167 & $17,949,532$ & $18,803,504$ & 853,972 & $92.3 \pm 23.4$ & \\
\hline \multirow{10}{*}{$\begin{array}{c}\text { Medical } \\
\text { departments }\end{array}$} & Internal Medicine & 8,957 & $2,298,155$ & $2,209,715$ & $\triangle 88,441$ & $99.8 \pm 15.2$ & \multirow{10}{*}{$\mathrm{F}=591.94 * *$} \\
\hline & Neurology & 879 & $2,371,328$ & $2,411,408$ & 40,080 & $94.2 \pm 12.8$ & \\
\hline & psychiatrics & 783 & $1,972,069$ & $2,230,084$ & 258,014 & $87.6 \pm 13.7$ & \\
\hline & General Surgery & 1,462 & $2,123,656$ & $2,588,742$ & 465,086 & $78.7 \pm 16.9$ & \\
\hline & Orthopedics & 2,386 & $3,094,224$ & $3,789,289$ & 695,064 & $76.2 \pm 16.4$ & \\
\hline & Neurosurgery & 999 & $3,953,971$ & $4,714,336$ & 760,365 & $82.8 \pm 13.6$ & \\
\hline & Cardiothoracic Surgery & 532 & $1,769,876$ & $2,099,688$ & 329,812 & $79.2 \pm 29.2$ & \\
\hline & Plastic Surgery & 201 & $1,092,740$ & $1,740,558$ & 647,818 & $55.2 \pm 23.5$ & \\
\hline & Ob\&Gy & 795 & 928,504 & $1,381,955$ & 453,451 & $73.2 \pm 31.3$ & \\
\hline & Pediatrics & 3,590 & 577,182 & 680,004 & 102,822 & $83.6 \pm 15.5$ & \\
\hline
\end{tabular}




\begin{tabular}{|c|c|c|c|c|c|c|c|}
\hline & ophthalmology & 235 & $1,096,738$ & $1,540,325$ & 443,587 & $81.5 \pm 50.0$ & \\
\hline & otolaryngology & 957 & 992,482 & $1,556,298$ & 563,816 & $69.8 \pm 29.1$ & \\
\hline & Urology & 891 & $1,306,660$ & $1,867,429$ & 560,769 & $74.5 \pm 20.3$ & \\
\hline & Rehabilitation & 298 & $6,098,281$ & $6,952,146$ & 853,865 & $84.8 \pm 24.3$ & \\
\hline & Family Medicine & 973 & $2,138,059$ & $2,051,413$ & $\triangle 86,646$ & $103.8 \pm 4.3$ & \\
\hline & Emergency Medicine & 574 & 561,555 & 708,295 & 146,740 & $72.1 \pm 18.0$ & \\
\hline \multirow[t]{2}{*}{ Operation } & Non-operation & 19,016 & $1,690,166$ & $1,720,076$ & 29,911 & $93.0 \pm 19.5$ & \multirow{2}{*}{$\mathrm{T}=5,646.85^{* *}$} \\
\hline & Operation & 5,630 & $3,056,834$ & $3,839,334$ & 782,500 & $71.1 \pm 18.1$ & \\
\hline \multirow{2}{*}{$\begin{array}{l}\text { Emergency } \\
\text { room visit }\end{array}$} & None visit & 15,971 & $1,938,687$ & $2,185,613$ & 246,927 & $89.1 \pm 22.6$ & \multirow{2}{*}{$\mathrm{T}=122.42 * *$} \\
\hline & Visit & 8,675 & $2,119,585$ & $2,238,385$ & 118,800 & $85.9 \pm 18.5$ & \\
\hline \multirow{3}{*}{$\begin{array}{c}\text { Classification } \\
\text { of DRG }\end{array}$} & Simple DRG & 3,818 & 843,322 & $1,054,517$ & 211,195 & $82.5 \pm 22.3$ & \multirow{3}{*}{$\mathrm{F}=316.77 * *$} \\
\hline & General DRG & 16,738 & $2,026,467$ & $2,236,780$ & 210,313 & $87.7 \pm 21.4$ & \\
\hline & Special DRG & 4,090 & $2,985,663$ & $3,144,022$ & 158,359 & $94.3 \pm 18.2$ & \\
\hline Total & Less than 510,000 & 5,658 & 366,559 & 534,078 & 167,519 & $77.2 \pm 23.2$ & \multirow{7}{*}{$\mathrm{F}=528.01 * *$} \\
\hline \multirow{6}{*}{$\begin{array}{c}\text { Medical costs } \\
\text { (Korean } \\
\text { Won) }\end{array}$} & $510,000-1,000,000$ & 6,787 & 724,788 & 904,132 & 179,344 & $85.8 \pm 20.5$ & \\
\hline & $1,010,000-1,500,000$ & 3,439 & $1,230,652$ & $1,423,338$ & 192,686 & $90.6 \pm 18.7$ & \\
\hline & $1,510,000-2,000,000$ & 2,173 & $1,745,122$ & $1,985,258$ & 240,136 & $92.7 \pm 19.2$ & \\
\hline & $2,010,000-2,5000,000$ & 1,286 & $2,248,346$ & $2,514,577$ & 266,232 & $93.5 \pm 18.3$ & \\
\hline & $2,510,000-5,000,000$ & 2,909 & $3,591,342$ & $3,873,826$ & 282,484 & $96.2 \pm 18.5$ & \\
\hline & More than $5,000,000$ & 2,394 & $8,769,460$ & $8,961,876$ & 192,417 & $98.7 \pm 15.7$ & \\
\hline & Total & 24,646 & $2,002,360$ & $2,204,188$ & 201,828 & $88.0 \pm 21.3$ & \\
\hline
\end{tabular}

1) The reason why Medical costs and Costs of Rehabilitation is high is that Average Care days of Rehabilitation patients(51.4 days) are much more than those of other departments(9.0 days)

2) $* \mathrm{p}<0.05, * * \mathrm{P}<0.01$

\subsection{Influence Factors on Cost Recovery Rate}

To find out the influence factors on Cost recovery rate, the study executed multiple regression analysis, taking Cost recovery rate into Dependent variable, and taking Inpatients Characteristics variables such as Gender, Age, Care days, Medical departments, Operation, Emergency room visit, DRG and the severity of DRG into Independent variables.

Medical departments were dummied on the basis of Psychiatry of which Cost recovery rate was most likely to approach the average Cost recovery rate of all Medical departments, and Emergency rooms and Operating rooms also were dummied by whether they were used or not. Moreover, The rate of 'Examination costs' and 'Physicians labor costs' was added because they were expected to influence much to Cost recovery rate.

As a consequence of multiple regression analysis, Cost recovery rate was influenced by Gender, Age, Medical departments, Emergency room visit, DRG, the severity of DRG, and the rate of Examination costs and Physicians labor costs. Among them, the rate of Examination costs and Physicians labor costs was most influential, and the Cost recovery rate went higher as Examination cost rate was high, and it went lower as Physicians labor cost rate was high $\langle$ Table 5>. 
Table 5. Influence Factor on Cost Recovery Rate

\begin{tabular}{|c|c|c|c|c|c|}
\hline \multirow[b]{2}{*}{ Independent variables } & \multicolumn{2}{|c|}{ Non-standardized } & \multirow[b]{2}{*}{$\begin{array}{c}\text { Standardized } \\
\text { regression } \\
\text { coefficient }\end{array}$} & \multirow[b]{2}{*}{ t-value } & \multirow[b]{2}{*}{$\begin{array}{l}\text { Significance } \\
\text { probability }\end{array}$} \\
\hline & $\begin{array}{l}\text { Regression } \\
\text { coefficient }\end{array}$ & $\begin{array}{l}\text { Standard } \\
\text { error }\end{array}$ & & & \\
\hline gender $(0=$ male $)$ & 0.006 & 0.002 & 0.012 & 2.620 & 0.009 \\
\hline Age & 0.000 & 0.000 & 0.015 & 2.016 & 0.044 \\
\hline Hospital visit days $§$ & 0.076 & 0.002 & 0.261 & 47.889 & 0.000 \\
\hline \multicolumn{6}{|l|}{$\begin{array}{l}\text { Medical } \\
(0=\text { Psychiatry })\end{array}$} \\
\hline Internal medicine & 0.055 & 0.007 & 0.098 & 7.329 & 0.000 \\
\hline Neurology & -0.011 & 0.010 & -0.008 & -1.147 & 0.251 \\
\hline General Surgery & 0.003 & 0.009 & 0.003 & 0.377 & 0.706 \\
\hline Orthopedics & 0.018 & 0.009 & 0.019 & 2.044 & 0.041 \\
\hline Neurosurgery & -0.065 & 0.009 & -0.048 & -6.905 & 0.000 \\
\hline Cardiothoracic Surgery & 0.084 & 0.012 & 0.041 & 7.279 & 0.000 \\
\hline Plastic Surgery & -0.129 & 0.019 & -0.033 & -6.811 & 0.000 \\
\hline Ob\&Gy & -0.116 & 0.010 & -0.077 & 11.921 & 0.000 \\
\hline Pediatrics & 0.009 & 0.008 & 0.012 & 1.123 & 0.262 \\
\hline ophthalmology & 0.185 & 0.017 & 0.056 & 11.086 & 0.000 \\
\hline otolaryngology & 0.021 & 0.010 & 0.013 & 2.099 & 0.036 \\
\hline Urology & -0.013 & 0.010 & -0.009 & -1.310 & 0.190 \\
\hline Rehabilitation & -0.132 & 0.013 & -0.053 & $\begin{array}{c}- \\
10.060\end{array}$ & 0.000 \\
\hline Family Medicine & 0.054 & 0.009 & 0.040 & 5.798 & 0.000 \\
\hline Emergency Medicine & -0.062 & 0.011 & -0.035 & -5.558 & 0.000 \\
\hline Other departments & 0.073 & 0.018 & 0.020 & 4.117 & 0.000 \\
\hline $\begin{array}{l}\text { Emergency room } \\
\text { visit( }(0=\text { None visit })\end{array}$ & -0.056 & 0.004 & -0.100 & 15.561 & 0.000 \\
\hline $\begin{array}{l}\text { Operation }(0=\text { None } \\
\text { operation })\end{array}$ & -0.002 & 0.006 & -0.003 & -0.374 & 0.708 \\
\hline DRG & 0.012 & 0.002 & 0.025 & 4.831 & 0.000 \\
\hline Examination cost rate§ & 0.107 & 0.002 & 0.406 & 53.056 & 0.000 \\
\hline Physician cost rate§ & -0.192 & 0.006 & -0.277 & $\begin{array}{c}- \\
30.097\end{array}$ & 0.000 \\
\hline (constant) & -0.461 & 0.017 & & 27.756 & 0.000 \\
\hline
\end{tabular}

1) $\mathrm{F}=1,087.50, \mathrm{df}=23,837, \mathrm{p}=0.000, \mathrm{R}^{2}=53.3 \%$

2) §: Hospital visit days and the rate of Examination costs and Physician costs are log converted values.

\section{Consideration and Conclusion}

This study analyzed In-patient costs in order to figure out Influence factors on Admission costs. The methods of resource use measurement can be classified into Bottom-up or Top-down, and Macro-costing or Micro-costing. That which method will be used is decided by the accuracy and the effectiveness of Cost accounting [11-13].

This study referred GDRG Cost analysis method which German InEK is carrying out, to calculate the accurate and efficient 'Patient cost'. Cost-centers were consisted of 10 and each cost-center was classified with Physician costs, Medical staff costs, Material costs, Equipment costs and Maintenance costs. 
When taking a look at Cost recovery rate by In-patient characteristics, it was high in the aged and for women. Also the longer admission days were, the lower Cost recovery rate got, which means that hospital benefits can be decreased if admission days go longer.

Cost recovery rate was finer when DRG and its severity were in more serious situation. Cost recovery of special DRG or the group with high severity was carried out better comparing to opposite groups, so it is expected that the possibility to reduce Medical service for the patients with high severity is low. However, the opposite interpretation is also possible. Provided that the patients are in General group or with low severity, it is possible for hospitals to decrease their services appealing their difficulties of benefit conservation. It may cause low Medical service quality and unintended health inequality.

Related to Medical departments, Cost recovery rate was low for Surgical units and high for Medical units. The problem was that Surgical units had high physician cost rate but Medical units had high examination cost rate. Also it can't be separated from social problems such as poor management due to the excessive physicians' labor costs, or such as the increasing examination cost abuse to cover up the insufficient medical benefit. This was confirmed in regression analysis in which the influence factors on Cost recovery rate were reviewed. The analysis showed that admission days, and the rate of examination costs and physicians'labor costs were very important influence factors on Cost recovery level. By the result of actual proof analysis, the cost recovery rate had a positive relationship with Examination costs and a negative relationship with physician costs.

This study is meaningful because it is an analysis connecting Health insurance claim data with Cost data for the first time in Korea. But it also has a few limits. First, the data for Cost analysis came from not many medical centers but a general hospital. Especially, the analysis input patients were only 24,646 who occupied only $0.87 \%$ of total general hospitals'health insurance admission numbers in 2010. For the reason, it is difficult for Patient costs built up in this study to have representativeness.

Second, it didn't include the costs of special wards(Isolation ward, Sterile treatment room and Neonatal unit) which take up the most part among admission costs, when setting up Cost-center unit. But there are the same limits in Germany where Cost analysis is very developed. Germany also calculates costs for only 3 units; General wards, Intensive care units and Delivery wards, and has been trying to improve Patient cost analysis method to make up for such a kind of problem for a long time.

Third, an hospital's uninsured benefits were not included in Cost recovery rate analysis, though $21.0 \%$ of Korea's total medical expenditure was uninsured in 2010[14]. That's because it's possible to calculate the total uninsured medical expenditures, but still difficult to calculate each patient's. If the data of uninsured medical expenditure is included, it is expected that the cost recovery rate will go up considerably.

It will help build up the reasonable medical insurance fee system, if Cost analysis for Out-patients is done, with making up for such limits. Moreover, plans for the objective data securing, well organized Cost analysis system and the accumulated data building up, based on advanced countries' experiences should be completed. Especially the government ought to set up the supporting system for medical service suppliers to intend to do those things.

\section{References}

[1] C. H. William, B. Peter, D. Daniel and R. B. Edmund, "Resource-Based Relative Values An Overview", JAMA., vol. 260, no. 16, (1988), pp. 2347-2353.

[2] Y. Na and K. H. Choi, "Case study of ABC build up with Hospital Cost system for a strategic decision making support", The Journal of Business Education., vol. 24, no. 3, (2010), pp. 387-413.

[3] S. S. Tan, L. Serden, A. Geissler, M. V, Ineveld, K. Redekop, M. Heurgren and L. H. Roijen, "Diagnosis-Related Groups in Europe", McGraw-Hill/Open University Press, (2011), pp. 59-74.

[4] C. St-Hilaire and P. K. Crepeau, "Hospital and unit cost-allocation methods", Healthcare Management Forum, vol. 13, (2000), pp. 12-32. 
[5] S. S. Tan, F. H. Rutten, M. V. Ineveld, K. Redekop and L. H. Roijen, "Comparing methodologies for the cost estimation of hospital services", The European Journal of Health Economics., vol. 20, no. 1, (2009), pp. 39-45.

[6] M. Vogl, "Improving patient-level costing in the English and the German 'DRG' system", Health Policy, vol. 109, no. 3, (2013), pp. 290-300.

[7] R. Busse, A. Geissler, W. Quentin and M. W. Wiley, "Diagnosis-Related Groups in Europe", McGrawHill/Open University Press, (2011), pp. 91-102.

[8] R. Busse, J. Schreyogg and P. C. Smith, "Editorial: hospital case payment systems in Europe", Health Care Management Science., vol. 9, (2006), pp. 211-213.

[9] M. H. Kim, "System buildup of cost-accounting by service and study of cost analysis", Korean Association of Tax and Accounting, vol. 2000, no. 1, (2000), pp. 99-119.

[10] Cost Accounting Standards Board, "Cost Accounting Standards Board: Restatement of Objectives, Policies and Concepts", (1977).

[11] W. Brouwer, F. Rutten and M. Koopmanschap, "Costing in Economic Evaluations: Economic Evaluation in Healthcare: Merging Theory with Practice", Oxford University Press, New York, (2001).

[12] D. B. Petitti, "Meta-Analysis, Decision Analysis, and Cost-Effectiveness Analysis: Methods for Quantitative Synthesis in Medicine", Oxford University Press, New York, (2000).

[13] M. Vogl, "Assessing DRG cost accounting with respect to resource allocation and tariff calculation: the case of Germany", Health Economics Review, vol. 2, no. 1, (2012), pp. 2-15.

[14] Organization for Economic Co-operation and Development, OECD Health Data 2013, (2014).

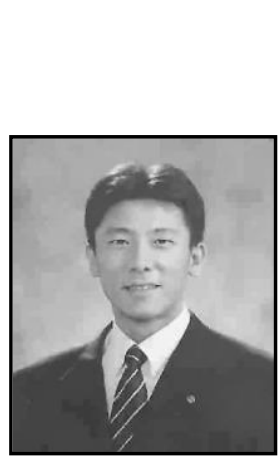

\section{Authors}

Woo Jung Sik, Assistant Professor / Ph.D. Research direction: Healthcare Management, Hospital Financial Management.

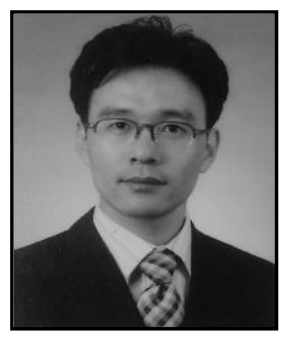

Kim Han Sung, Assistant Professor / Ph.D. Research direction: Healthcare Management, Hospital Cost Analysis. 